\title{
RETENCIÓN DE HUEVOS EN HEMBRA DE JABOTÍ -PIRANGA (Geochelone carbonaria - Spix, 1824) EN CAUTIVERIO
}

\author{
EGGS RETENTI ON I N FEMALE OF “J ABOTY-PI RANGA" \\ (Geochelone carbonaria - Spix, 1824) I N CAPTI VI TY
}

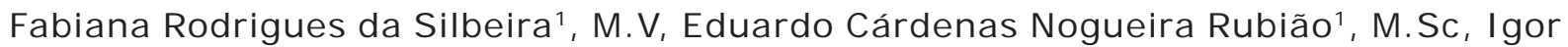
Mansur Muniz ${ }^{1}$, M.Sc, Denise de Mello Bobány ${ }^{1 *}$, M.Sc.

\begin{abstract}
${ }^{1}$ Centro Universitário Serra dos Órgãos - Curso de Graduação em Medicina Veterinária, Estrada da Prata s/n - campus Quinta do Paraíso, Teresópolis, RJ, Brazil. *Correspondência: debobany@gmail.com
\end{abstract}

Recibido: Octubre 31 de 2008; Aceptado: Julio 30 de 2009

\section{RESUMEN}

Un J abotí-Piranga adulto (Geochelone carbonaria), hembra, con peso de 3.5 kg ingresó por consulta en la escuela clínica "Dr. Cataldi Luiz de Souza", del "Centro Universitário Serra dos Órgãos - Teresopolis, Río de Janeiro", con historia de retención de huevos. En la evaluación clínica y radiológica, se encontró tres huevos en posiciones normales. Teniendo en cuenta la temporada de reproducción, se aconsejó la observación del animal. Después de un año, dicho animal regresó a la clínica por no haber todavía expulsado los huevos. Tras nuevo examen radiográfico se observó que los 3 huevos continuaban en la misma posición. A continuación, se aplicó $0.03 \mathrm{ml}$ de oxitocina por vía subcutanea ( $10 \mathrm{Ul}$ x ml), en el apéndice anterior del animal. Tres horas después de la aplicación de la hormona, el animal expulsó los huevos naturalmente, sin la necesidad de una intervención quirúrgica, con lo que se puede sospechar de la eficacia de la aplicación de oxitocina para este procedimiento en jabotí Piranga.

Palabras clave: Jaboti, Geochelone carbonaria, retención de huevos, oxitocina.

\section{ABSTRACT}

An adult specimen of "J aboty-piranga" (Geochelone carbonaria), female, weighing $3.5 \mathrm{~kg}$, was attended in the Doctor-school Dr. Luiz Cataldi de Souza of the University Centre Serra dos Órgãos - Teresópolis, Rio de Janeiro, presenting historically of eggs retention. By means of physical and radiografic examination the presence of three eggs was noted in normal positions. Because of being a reproductive station, the observation of the animal was advised to the owner, a year later, the tortoise was returned to the clinic because of 
not having expelled the eggs. After new radiografic examination, the presence of three eggs in the same positions was diagnosed, and $0.03 \mathrm{~mL}$ of oxitocine was applied, for subcutaneous road in the previous appendix of the animal. Passed three hours of the application of the hormone, the female of giant tortoise-pauper expelled the eggs naturally, no surgical intervention was needed.

Key words: Jaboti, Geochelone carbonaria, egg retention, oxitocine.

\section{NTRODUCCIÓN}

El “Jabotí-piranga", superfamilia Testudinoidea, familia Testudinidae, especie Geochelone carbonaria es una tortuga terrestre con escalas de color rojo en la cabeza y en los pies y una amplia distribución geográfica en toda América del Sur, creado mucho en cautiverio $(1,2)$.

Las tortugas presentan características diferentes que dificultan su mantenimiento en cautividad como animales de compañía, por ejemplo: son animales ectotérmicos, y con los hábitos diurnos, su temperatura de confort varía de 25 a $30^{\circ} \mathrm{C}(3,4)$, son oviparous y sus huevos son retenidos en el oviducto por un período de 1 a 2 semanas para formar la cáscara (5). Para la puesta de los huevos, las hembras buscan un terreno de topografía llana y tierra suelta, preferentemente de arena para facilitar la excavación del agujero para la depositar los huevos. Cuando el medio ambiente en que viven no ofrece las condiciones ideales para ovopostura, puede ocurrir retención de los huevos, lo que ocurre a menudo bajo las condiciones de cautiverio (3-5).

Además, las tortugas necesitan de cuidados especiales con la nutrición, evitando los restos de la alimentación humana o alimentos, pues, al momento de suministrárselos, pueden no estar frescos y ocasionar deficiencias de vitaminas o debilidades diversas, que predisponen a la retención de los huevos $(6,7)$.

Es evidente que otros factores como la cáscara rota del huevo, excremento resecado, traumatismos, trastornos endocrinos, anomalías del fotoperiodo, entre otros, también pueden causar retención de los huevos (3).
Cuando esto sucede, las clínicas veterinarias utilizan principalmente el tratamiento clínico con la estimulación hormonal, o sea, oxitocina en dosis de 1 a $10 \mathrm{UI} / \mathrm{kg}$ de peso, aplicada por vía subcutánea (SC) en el apéndice anterior, hasta tres dosis, a intervalos de 24 horas (8). Si la hembra se encuentra agotada, es prudente inyectar gluconato de calcio (150 a $300 \mathrm{mg} / \mathrm{kg}$ ) también por via SC o IC alrededor de 30 60 minutos antes de la administración de la hormona (2).

Si el tratamiento clínico no tiene éxito, el tratamiento quirúrgico será el indicado para este animal (4).

El objetivo de este caso clínico fue establecer una referencia para el tratamiento clínico de la retención de huevos en una tortuga destinada al mercado de mascotas.

\section{HI STORI A CLÍ NI CA}

Un ejemplar hembra de Piranga-Jaboti (Geochelone carbonaria) con domicilio en la ciudad de Teresópolis, Rio de J aneiro, Brasil, con $36 \mathrm{~cm}$ de largo del carapacho y $3.5 \mathrm{~kg}$ fue examinado en la Clínica de la Escuela de Veterinaria Dr. Cataldi Luiz de Souza, Teresopolis, Río de Janeiro, con una fuerte indicación de retención de huevos en la cavidad por cavar hoyos en el jardín de la vivienda que, en este caso, consistió en áreas de superficie dura con tierra como sustrato esporádico.

La ciudad de Teresópolis, en Río de Janeiro, Brasil se encuentra a $22^{\circ} 25^{\prime}$ - $22^{\circ} 32^{\prime} \mathrm{S}$ y entre $42^{\circ} 59^{\prime}$ e $43^{\circ} 07^{\prime} \mathrm{W}$. El clima, de acuerdo con el "Instituto Brasileiro de Geografia e 
Estadística (IBGE), es tropical, caluroso y húmedo, altitud de $1650 \mathrm{~m}$, con precipitación media anual de $900 \mathrm{~mm}$ y promedio de temperatura anual de $20^{\circ} \mathrm{C}$, con un máximo de $25^{\circ} \mathrm{C}$ y un mínimo de $12^{\circ} \mathrm{C}$.

Según el propietario, el animal se alimentaba de frutas y hortalizas y, a veces, con un alimento o pienso concentrado para perros que, en Brasil, según la Normativa № 09 del 09 de Julio del 2003, del "Ministério da Agricultura, Pecuária e Abastecimento", presentan promedio, 16 a 18\% de proteina, 4.5 a $6.0 \%$ de glúcidos y 12 a $30 \%$ de humedad.

Al paciente se le realizó una radiografía en proyección ventro dorsal en la que se identificó la presencia de tres huevos. Por estar en el período reproductivo, si aconsejó observar el animal para que se diera la oviposición natural.

Después de un año, el propietario regresó con el animal a la clínica, puesto que el jabotí no había cavado más en su jardín y todavía no había realizado la postura de los huevos.

Se repitieron las radiografías y se han detectado, una vez mas, los tres huevos. Cuando se las comparó con las primeras radiografías, se concluyó que los tres huevos todavía estaban en la misma posición, aúnque en el segundo examen los huevos parecían estar más calcificados (Figuras 1 y 2).

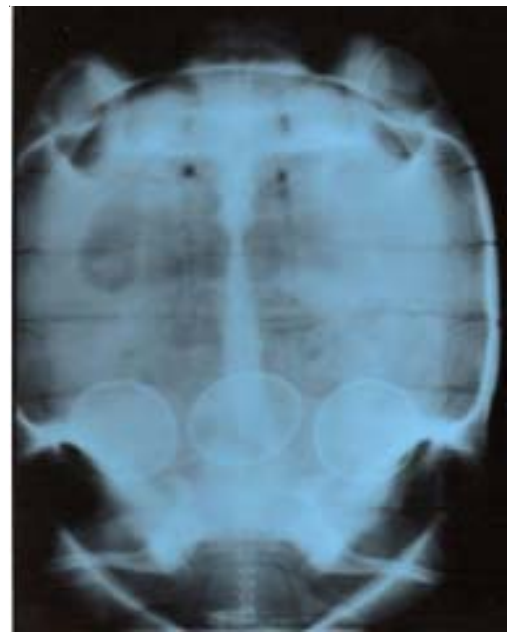

Figura 1. Radiografia, celebrada el $28 / 04 / 2006$

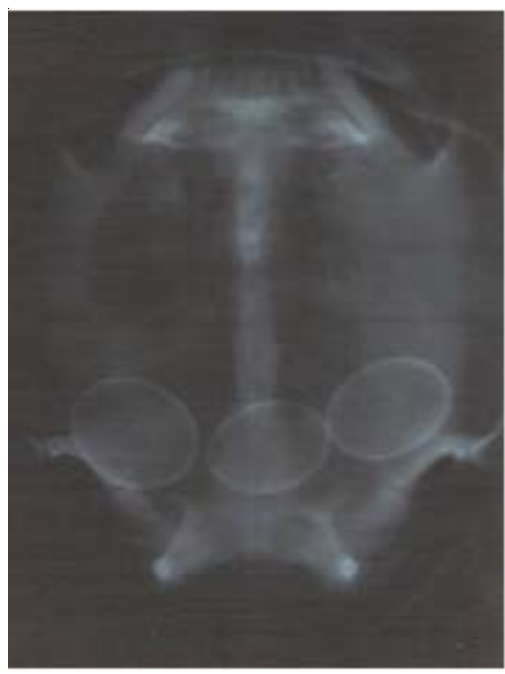

Figura 2. Radiografia, celebrada el 09/03/2007

Por lo tanto, se decidió realizar el tratamiento clínico con la aplicación de $0.03 \mathrm{ml}$ de oxitocina ( $10 \mathrm{Ul}$ x ml) vía subcutánea en el miembro anterior del animal (Figura 3).

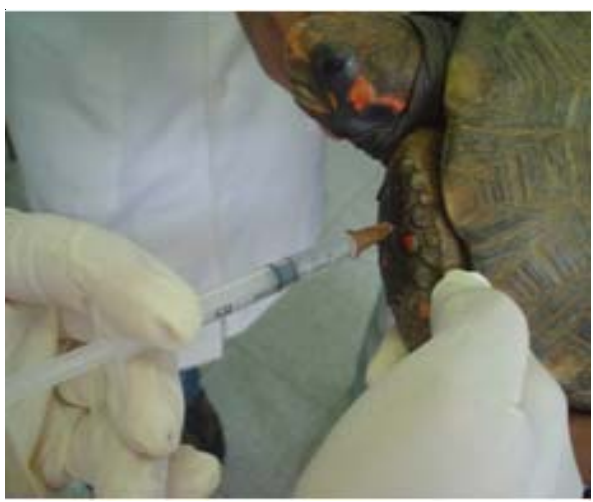

Figura 3. Aplicación de oxitocina en el apéndice anterior de la hembra de J abotí-Piranga.

Después de tres horas de aplicación de la hormona, la hembra de J abuti-Piranga expulsó a los huevos sin ningún problema (Figura 4).

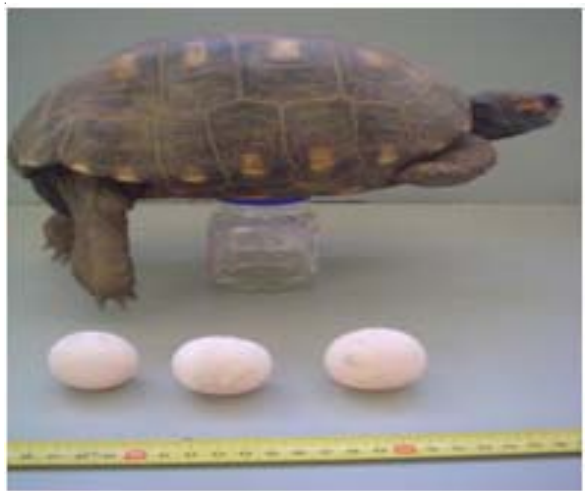

Figura 4. Hembra de J abotí-Piranga con los huevos expulsados. 


\section{DISCUSIÓN}

En este caso, algunos factores pueden haber contribuido a la retención de los huevos por más de un año, tales como medio ambiente inadecuado (clima, humedad y sustrato) con suministro alimentar irregulares $(6,7)$. Esto porque el suministro de una dieta desequilibrada, juntamente con el mantenimiento de las tortugas en zonas con predominio de suelo duro, es determinante para la aparición de retención de huevos (3). Hecho que refuerza la importancia de un correcto manejo y comprensión de la correcta gestión antes de se adquirir una tortuga como mascota, evitando futuras complicaciones (3-5-7).
En caso de sospecha de retención de huevos, el uso de la radiografía es fundamental, tanto para el diagnóstico como para la decisión de se realizar el tratamiento con oxitocina $(2,4,8)$ que, en este caso, fue eficaz para promover la expulsión de los huevos después de la primera dosis de la hormona $(2,8)$.

Ante esta situación, es evidente que la selección de un animal de compañía debe hacerse entre los animales domésticos. Esto debido al comprometimiento del bienestar de los animales salvajes que viven en cautiverio, lo que puede contribuir al aumento de la morbilidad y mortalidad de estos animales.

\section{REFERENCI AS}

1. Klingenberg RJ. Animais Exóticos e Laboratoriais: Répteis In Aiello SE, Mays A. Manual Merck de Veterinária. 8aed. São Paulo: Roca; 2001.

2. Goulart SEC. Herpentologia, Herpetocultura e Medicina de Répteis. $1^{\circ}$ ed. Rio de J aneiro: LF livros; 2004.

3. Matias CAR, Romão MAP, Tortelly, R, Bruno, SF. Aspectos fisiopatológicos da retenção de ovos em J abutipiranga (Geochelone carbonaria Spix, 1824). Cienc Rural 2006; 36(5): 1494- 1500.

4. Cubas HP, Baptistotte C. Anatomia e Fisiologia. In Cubas SZ, Silva RCJ, Dias C LJ . Tratado de Animais Selvagens -

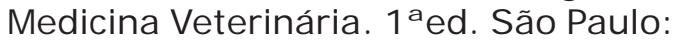
Roca; 2007.

5. Matias CAR. Aspectos Fisiopatológicos da Retenção de Ovos em jabutipiranga (Geochelone carbonaria Spix, 1824) Mantidos como Animais de Companhia. (Dissertação de Mestrado na Área de Concentração: Reprodução e Inseminação Artificial). Niterói: Universidade Federal Fluminense; 2003.
6. Divers HMS, Divers HJ S. Quelônios. Em: Aguilar R, Divers HMS, Divers HJS.. Atlas de Medicina, Terapêutica e Patologia de Animais Exóticos. $1^{\circ}$ ed. São Caetano do Sul: Inter-Book; 2007.

7. Hernández OE. Reproducción y Crecimiento del Morrocoy, Geochelone (Chelonoidis) carbonaria (Spix, 1824) (Reptilia, Testudinidae). BIOLLANIA 1997; 13: 165-183.

8. Oliveira AMP. Animais Silvestres e Exóticos na Clínica Particular. $1^{\circ}$ ed. São Paulo: Roca; 2003. 